\title{
THE DISTRIBUTION OF SEPARATIONS OF DMSA HIPPARCOS CATALOGUE
}

\author{
J. F. Ling,,$^{1,2}$ P. Magdalena, ${ }^{1,3}$ and C. Prieto ${ }^{1,3}$ \\ RESUMEN
}

Se han construído muestras de volumen limitado para las binarias abiertas del Anexo de Estrellas Dobles y Múltiples del Catálogo Hipparcos (DMSA, Sección C) hasta distancias de 100 pc y 200 pc. Hemos estudiado la distribución de las separaciones lineales para dichas muestras. Se ha encontrado que se ajustan a una distribución muy próxima a la distribución de Öpik para separaciones comprendidas entre 10 y 800 UA (muestra de $100 \mathrm{pc}$ ) y entre 15 y $1400 \mathrm{UA}$ (muestra de $200 \mathrm{pc}$ ).

\section{ABSTRACT}

We have constructed volume-limited samples of wide binaries in the Hipparcos Catalogue Double and Multiple Systems Annex (DMSA, Section C), out to distances of $100 \mathrm{pc}$ and $200 \mathrm{pc}$. We study the distribution of linear separations for these samples of binaries. We find that they closely follow Öpik's distribution in the interval of separations between about 10 and $800 \mathrm{AU}$ (for the $100 \mathrm{pc}$ sample), and between 15 and $1400 \mathrm{AU}$ (for the 200 pc sample)

\section{Key Words: BINARIES: GENERAL}

The current studies about the distribution of separations between components in wide binaries of different catalogues and stellar groups are diverse. (Poveda, 1988; Poveda, Allen \& Herrera, 1997, 2000; Allen, Poveda \& Herrera 1997; Herrera et al. 1997). As is well known, the importance of these investigations resides in the information provided by the distribution of the different elements of the binaries about the initial conditions and processes involved in their formation and subsequent dynamical evolution.

In this sense, we want to make use of the results obtained by the first astrometric space mission made by the Hipparcos satellite, and published in 1997 in the Hipparcos and Tycho Catalogues (ESA, 1997), so as to undertake a study of the same characteristics as in the aforementioned work for the binaries that were observed by the satellite. In fact, the homogeneity, the precision in the measurements of both position and trigonometric parallaxes (milliarcsecond accuracy), make this catalogue an appropriate framework for continued testing of the hypotheses which are proposed in these same articles.

In order to carry out a reliable study on a set of elements, referring as much to the astrometric pa-

\footnotetext{
${ }^{1}$ Observatorio Astronómico R. M. Aller, Universidade de Santiago de Compostela, Santiago de Compostela, Spain. email: oafana@usc.es

${ }^{2}$ Dpto. Matemática Aplicada, Universidade de Santiago de Compostela, Santiago de Compostela, Spain.

${ }^{3}$ Dpto. Matemática Aplicada II, Universidade de Vigo, Vigo, Spain.
}

rameters determined by the Hipparcos satellite as to the physical relation between the components of the binaries, as well as taking into account the completeness of the Hipparcos catalogue (Söderhjelm, 2000), we have established the following selection criteria:

- The binaries of the catalogue which the satellite has been able to resolve into their distinct components, are considered (Section $\mathrm{C}$ of the Hipparcos Catalogue Double and Multiple Systems Annex (DMSA).)

- Of these, we discard those which either have negative parallaxes, which may arise when the true parallax is smaller than its error, or form optical pairs, where the proper motions of each component differ greatly in magnitude or direction.

- As a simplified approximation, it is assumed that the Hipparcos catalogue is complete up to visúal magnitude 7.3 .

Once the previous guidelines have been established, one works with volume-limited samples. So by fixing a value for the distance, the extraction of the samples is carried out, keeping only stars brighter than the absolute visual magnitude limit $M_{v}$ given by $M_{v}=m_{v}+5+5 \log \Pi$, where $m_{v}=7.3$, the apparent visual magnitude which establishes the completeness of the Hipparcos Catalogue and $\Pi$ is the parallax determined by the satellite in arcseconds. 
The resulting sample is uniformly selected in the sense of the gradient in number density of binaries with distance, because the flux limit is no longer present, thereby minimizing the possible selection effects. This allows us to deal with sets with a reasonable degree of certainty, in what is referred to as the statistical nature of the work.

In this case, taking into account the Hipparcos photometric system, work has been done with the median magnitude $H_{p}$ of the binaries, so as to form two samples, both of which are situated in the solar vicinity; one corresponding to a sphere centred on the mean position of the Hipparcos satellite for 1991.25 and with a radius of 100 pcs and the other a radius of 200 pcs. The characteristics of the sample with distances less than 100 pcs are: absolute visual magnitude limit $M_{v}=2.30$, the primary and the secondary components have absolute visual magnitudes which are brighter than 2.30 , the magnitude difference between the components varies between $0.019 \leq$ $\Delta H_{p} \leq 3.389$, the trigonometric parallaxes vary between $10.06 \leq \Pi($ mas $) \leq 63.27$ and the total number of binaries is 58 . The corresponding characteristics of the sample with distances less than 200 pcs are: absolute visual magnitude limit $M_{v}=0.80$, the primary and the secondary components have absolute visual magnitudes which are brighter than 0.80 , the magnitude difference between the components varies between $0.002 \leq \Delta H_{p} \leq 3.389$, the trigonometric parallaxes vary between $5.16 \leq \Pi($ mas $) \leq 25.96$ and the total number of binaries is 56 . It is noticed that the number of elements in the second sample is less than in that of the first sample, due to the strong restriction that implies passing from $M_{v}=2.30$ to $M_{v}=0.80$.

The behaviour of the distribution of the semimajor axes $(a)$ of our binaries shall be studied through their projected separations $(s)$ in astronomical units, now that we have the benefit of knowing the values of their parallaxes. For this purpose we shall use the methodology utilized by Poveda et al. $(1997,2000)$ understanding that distributions used by Poveda will be equivalent; on assuming the following theoretical relation, obtained by Couteau in 1960, between the expectation values of the logarithms of both parameters $E(\log a)-E(\log s)=0.146$.

If we compare the graphic representation of the cumulative distribution of the logarithms of the separations $N(\log s)$ versus $\log s$ in an orthogonal system, binary by binary, for the total number of elements of the sample, with Öpik's relation (1924) $f(s) \propto 1 / s$, given by a straight line, it is observed that the best linear fit of the data corresponds to the part of the line situated between a certain interval of values of $s$. In such a way one clearly notices that from certain separations the data begin to depart from the said relation.

The upper limits $800 \mathrm{AU}$ and $1400 \mathrm{AU}$ for each one of the respective samples, also seem to be related to instrumental limitations of the Hipparcos satellite's main detector, where only the signal for systems $0.1 \leq \rho \leq 10 \operatorname{arcsec}$ and $\Delta H_{p} \leq 4 \mathrm{mag}$ could be decomposed into the contributions of the different stellar components.

Even though the statistic utilized is somewhat limited, it has been verified that in the volume sample (limited to distances of both 100 pcs and 200 pcs), the behaviour of wide binary stars observed by the Hipparcos satellite conforms to an "Öpik" type distribution. Both sets of binaries of the solar vicinity seem to confirm the thesis that for binaries with $a \geq 40$ AU Öpik's distribution is the most appropriate, as opposed to Kuiper's Gaussian distribution (Poveda et al. 2000).

We would like to express our thanks to Drs. A. Poveda and C. Allen for their invaluable help and advice, without which this work would not have been possible. This work was supported by the Xunta de Galicia (Spain) under the project PGIDIT02PXIC 24301PN

\section{REFERENCES}

Allen, C., Poveda, A. \& Herrera, M. A., 1997, in Visual Double Stars: Formation, Dynamics and Evolutionary Tracks, ed. J. A. Docobo, A. Elipe \& H. McAlister (Dordrecht: Kluwer), 133

Couteau, P., 1960, J. des Observateurs, 43, 41

ESA, 1997, The Hipparcos and Tycho Catalogues, ESASP-1200 (Noordwijk; ESA)

Herrera, M. A., Poveda, A., Nigoche, A. and Segura, A., 1997, in Visual Double Stars: Formation, Dynamics and Evolutionary Tracks, ed. J. A. Docobo, A. Elipe \& H. McAlister (Dordrecht: Kluwer), 199

Öpik, E. J., 1924, Tartu Obs. Publ., 25, No. 6

Poveda, A., 1988, Ap\&SS, 142, 67

Poveda, A., Allen, C. and Herrera, M. A., 1997, in Visual Double Stars: Formation, Dynamics and Evolutionary Tracks, ed. J. A. Docobo, A. Elipe \& H. McAlister (Dordrecht: Kluwer), 191

Poveda, A., Allen, C. and Herrera, M. A. 2000, in Stellar Astrophysics, ed. K. S. Cheng, H. F. Chau, K. L. Chan \& K. C. Leung (Dordrecht: Kluwer), 181

Söderhjelm, S., 2000, AN, 321, 165 